YITP-00-36

\title{
Gravity in Randall-Sundrum Brane World Revisited
}

\author{
Zurab Kakushadze* \\ C.N. Yang Institute for Theoretical Physics \\ State University of New York, Stony Brook, NY 11794
}

(August 15, 2000)

\begin{abstract}
We point out some subtleties with gauge fixings (which sometimes include the so-called "brane bending" effects) typically used to compute the graviton propagator on the Randall-Sundrum brane. In particular, the brane, which has non-vanishing tension, explicitly breaks some part of the diffeomorphisms, so that there are subtleties arising in going to, say, the axial gauge or the harmonic gauge in the presence of (non-conformal) matter localized on the brane. We therefore compute the graviton propagator in the gauge where only the graviphoton fluctuations are set to zero (the diffeomorphisms necessary for this gauge fixing are intact), but the graviscalar component is untouched. We point out that in the Gaussian normal coordinates (where the graviscalar component vanishes on the brane) the graviton propagator blows up in the ultra-violet near the brane. In fact, the allowed gauge transformations, which do not lead to such ultra-violet behavior of the graviton propagator, are such that the coupling of the graviscalar to the brane matter cannot be gauged away in the ultra-violet. Because of this, at the quantum level, where we expect various additional terms to be generated in the brane world-volume action including those involving the graviscalar, fine-tuning (which is independent of that for the brane cosmological constant) is generically required to preserve consistent coupling between bulk gravity and brane matter. We also reiterate that in such warped backgrounds higher curvature terms in the bulk are generically expected to delocalize gravity.
\end{abstract}

Typeset using REVTEX

*E-mail: zurab@insti.physics.sunysb.edu 


\section{THE MODEL}

In the Brane World scenario the Standard Model gauge and matter fields are assumed to be localized on branes (or an intersection thereof), while gravity lives in a larger dimensional bulk of space-time [1-12]. The volume of dimensions transverse to the branes is automatically finite if these dimensions are compact. On the other hand, the volume of the transverse dimensions can be finite even if the latter are non-compact. In particular, this can be achieved by using [13] warped compactifications [14] which localize gravity on the brane. A concrete realization of this idea was given in [15].

In this paper we study gravitational interactions between matter sources localized on the brane in the Randall-Sundrum model, whose action is given by:

$$
S=-f \int_{\Sigma} d^{D-1} x \sqrt{-\widehat{G}}+M_{P}^{D-2} \int d^{D} x \sqrt{-G}[R-\Lambda] .
$$

For calculational convenience we will keep the number of space-time dimensions $D$ unspecified. In (11) $M_{P}$ is the $D$-dimensional (reduced) Planck scale, while $\Lambda$ is the bulk vacuum energy density, which is assumed to be a negative constant. The $(D-1)$-dimensional hypersurface $\Sigma$, that is, the brane, is the $z=0$ slice of the $D$-dimensional space-time, where $z \equiv x^{D}$. Next,

$$
\left.\widehat{G}_{\mu \nu} \equiv \delta_{\mu}{ }^{M} \delta_{\nu}{ }^{N} G_{M N}\right|_{z=0}
$$

where the capital Latin indices $M, N, \ldots=1, \ldots, D$, while the Greek indices $\mu, \nu, \ldots=$ $1, \ldots,(D-1)$. The quantity $f$ is the brane tension'?

The equations of motion following from the action (11) read:

$$
R_{M N}-\frac{1}{2} G_{M N} R+\frac{1}{2} G_{M N} \Lambda+\frac{1}{2} \frac{\sqrt{-\widehat{G}}}{\sqrt{-G}} \delta_{M}^{\mu} \delta_{N}{ }^{\nu} \widehat{G}_{\mu \nu} \tilde{f} \delta(z)=0
$$

where $\tilde{f} \equiv f / M_{P}^{D-2}$.

In the following we will be interested in solutions to the equations of motion following from the action (11) with the warped metric of the following form:

$$
d s_{D}^{2}=\exp (2 A) \eta_{M N} d x^{M} d x^{N}
$$

where $\eta_{M N}$ is the flat $D$-dimensional Minkowski metric, and the warp factor $A$, which is a function of $z$, is independent of the coordinates $x^{\mu}$. With this ansätz, we have the following equations of motion for $A$ (prime denotes derivative w.r.t. $z$ ):

\footnotetext{
${ }^{1}$ There might be additional bulk fields other than gravity. We will assume that such fields have vanishing expectation values. Also, there might be various fields localized on the brane. If the brane world-volume theory is not conformal, then, as was pointed out in [16,17], quantum loops of these fields are generically expected to induce, among other terms, the $(D-1)$ dimensional Einstein-Hilbert term on the brane. We will discuss the consequences of this at the end of the paper. For now, however, we will ignore any such terms and stick to the original action (1).
} 


$$
\begin{aligned}
& (D-1)(D-2)\left(A^{\prime}\right)^{2}+\Lambda \exp (2 A)=0, \\
& (D-2)\left[A^{\prime \prime}-\left(A^{\prime}\right)^{2}\right]=-\frac{1}{2} \exp (A) \tilde{f} \delta(z) .
\end{aligned}
$$

This system of equations has a solution if we fine-tune the brane tension to the bulk vacuum energy density as follows:

$$
\Lambda=-\frac{1}{16} \frac{D-1}{D-2} \widetilde{f}^{2}
$$

Assuming that the brane tension is positive, the solution is then given by

$$
A(z)=-\ln \left[\frac{|z|}{\Delta}+1\right]
$$

where $\Delta \equiv 4(D-2) / \widetilde{f}$, and we have chosen the integration constant such that $A(0)=0$.

The volume of the transverse dimension, which is given by $\int d z \exp (D A)$, is finite in the above solution, and so is the normalization of the $(D-1)$-dimensional graviton zero mode wave-function, which is given by $\int d z \exp [(D-2) A]$. That is, gravity is localized on the brane, and at long distances we expect that gravity is $(D-1)$-dimensional. In particular, at long distances we expect $(D-1)$-dimensional Newton's law to be valid for matter sources localized on the brane, and the number of the graviton degrees of freedom which couple to the corresponding conserved energy-momentum tensor on the brane should be $(D-1)(D-4) / 2$ (which for $D=5$ is 2). On the other hand, at short distances we expect that gravity is $D$-dimensional. In particular, at short distances we expect the $D$-dimensional Newton's law to take over. As to the tensor structure of graviton propagator, consistency tells us that we should also expect it to be that of the $D$-dimensional massless graviton propagator. The number of the graviton degrees of freedom which couple to the conserved energy-momentum tensor on the brane should then be $(D-2)(D-3) / 2=(D-1)(D-4) / 2+1$ (which for $D=5$ is 3 ) .

\section{BRANE WORLD GRAVITY}

To understand how such a smooth crossover between the number of relevant degrees of freedom occurs in this model, let us study small fluctuations around the solution:

$$
G_{M N}=\exp (2 A)\left[\eta_{M N}+\widetilde{h}_{M N}\right]
$$

where for convenience reasons we have chosen to work with $\widetilde{h}_{M N}$ instead of metric fluctuations $h_{M N}=\exp (2 A) \widetilde{h}_{M N}$.

\footnotetext{
${ }^{2}$ Note that the other $D-3=D(D-3) / 2-(D-2)(D-3) / 2$ degrees of freedom (that is, those corresponding to the graviphoton) do not couple to the conserved energy-momentum tensor on the brane.
} 
To proceed further, we need equations of motion for $\widetilde{h}_{M N}$. Let us assume that we have matter localized on the brane, and let the corresponding conserved energy-momentum tensor be $T_{\mu \nu}$ :

$$
\partial^{\mu} T_{\mu \nu}=0
$$

The graviton field $\widetilde{h}_{\mu \nu}$ couples to $T_{\mu \nu}$ via the following term in the action (note that $\widetilde{h}_{\mu \nu}=h_{\mu \nu}$ at $z=0$ as we have set $A(0)=0)$ :

$$
S_{\mathrm{int}}=\frac{1}{2} \int_{\Sigma} d^{D-1} x T_{\mu \nu} \widetilde{h}^{\mu \nu}
$$

Next, starting from the action $S+S_{\text {int }}$ we obtain the following linearized equations of motion for $\widetilde{h}_{M N}$ (the capital Latin indices $M, N, \ldots$ are lowered and raised with the flat $D$-dimensional Minkowski metric $\eta_{M N}$ and its inverse):

$$
\begin{aligned}
& \left\{\partial_{S} \partial^{S} \widetilde{h}_{M N}+\partial_{M} \partial_{N} \widetilde{h}-\partial_{M} \partial^{S} \widetilde{h}_{S N}-\partial_{N} \partial^{S} \widetilde{h}_{S M}-\eta_{M N}\left[\partial_{S} \partial^{S} \widetilde{h}_{-} \partial^{S} \partial^{R} \widetilde{h}_{S R}\right]\right\}+ \\
& (D-2) A^{\prime}\left\{\left[\partial_{S} \widetilde{h}_{M N}-\partial_{M} \widetilde{h}_{N S}-\partial_{N} \widetilde{h}_{M S}\right] n^{S}+\eta_{M N}\left[2 \partial^{R} \widetilde{h}_{R S}-\partial_{S} \widetilde{h}\right] n^{S}\right\}+ \\
& (D-1)(D-2)\left(A^{\prime}\right)^{2} \eta_{M N} \widetilde{h}_{S R} n^{S} n^{R}=-M_{P}^{2-D} \widetilde{T}_{M N} \delta(z),
\end{aligned}
$$

where $\widetilde{h} \equiv \widetilde{h}_{M}^{M}$, and for notational convenience we have introduced a unit vector $n^{M}$ with the following components: $n^{\mu}=0, n^{D}=1$. The components of $\widetilde{T}_{M N}$ are given by:

$$
\begin{aligned}
& \widetilde{T}_{\mu \nu} \equiv T_{\mu \nu}-\frac{1}{2} \eta_{\mu \nu} f \rho, \\
& \widetilde{T}_{\mu \nu}=A_{\mu} f \\
& \widetilde{T}_{D D}=0
\end{aligned}
$$

Here and in the following we use the notations:

$$
H_{\mu \nu} \equiv \widetilde{h}_{\mu \nu}, \quad A_{\mu} \equiv \widetilde{h}_{\mu D}, \quad \rho \equiv \widetilde{h}_{D D} .
$$

Also, we will use the notation $H \equiv H_{\mu}^{\mu}$, and the Greek indices $\mu, \nu, \ldots$ are lowered and raised with the flat $(D-1)$-dimensional Minkowski metric $\eta_{\mu \nu}$ and its inverse.

The above equations of motion are invariant under certain gauge transformations corresponding to unbroken diffeomorphisms. In terms of $\widetilde{h}_{M N}$ the full $D$-dimensional diffeomorphisms

$$
\delta h_{M N}=\nabla_{M} \xi_{N}+\nabla_{N} \xi_{M}
$$

are given by the following gauge transformations (here we use $\xi_{M} \equiv \exp (2 A) \widetilde{\xi}_{M}$ ):

$$
\delta \widetilde{h}_{M N}=\partial_{M} \widetilde{\xi}_{N}+\partial_{N} \widetilde{\xi}_{M}+2 A^{\prime} \eta_{M N} \widetilde{\xi}_{S} n^{S}
$$

Note, however, that the presence of the brane (whose tension is non-vanishing) breaks the full $D$-dimensional diffeomorphism invariance (18) to a smaller subset of gauge transformations. To deduce the unbroken gauge transformations, let us first rewrite the equations of motion (12) in terms of the components: 


$$
\begin{aligned}
& \left\{\partial_{\sigma} \partial^{\sigma} H_{\mu \nu}+\partial_{\mu} \partial_{\nu} H-\partial_{\mu} \partial^{\sigma} H_{\sigma \nu}-\partial_{\nu} \partial^{\sigma} H_{\sigma \mu}-\eta_{\mu \nu}\left[\partial_{\sigma} \partial^{\sigma} H-\partial^{\sigma} \partial^{\rho} H_{\sigma \rho}\right]\right\}+ \\
& \left\{H_{\mu \nu}^{\prime \prime}-\eta_{\mu \nu} H^{\prime \prime}+(D-2) A^{\prime}\left[H_{\mu \nu}^{\prime}-\eta_{\mu \nu} H^{\prime}\right]\right\}- \\
& \left\{\partial_{\mu} A_{\nu}^{\prime}+\partial_{\nu} A_{\mu}^{\prime}-2 \eta_{\mu \nu} \partial^{\sigma} A_{\sigma}^{\prime}+(D-2) A^{\prime}\left[\partial_{\mu} A_{\nu}+\partial_{\nu} A_{\mu}-2 \eta_{\mu \nu} \partial^{\sigma} A_{\sigma}\right]\right\}+ \\
& \left\{\partial_{\mu} \partial_{\nu} \rho-\eta_{\mu \nu} \partial_{\sigma} \partial^{\sigma} \rho+\eta_{\mu \nu}\left[(D-2) A^{\prime} \rho^{\prime}+(D-1)(D-2)\left(A^{\prime}\right)^{2} \rho\right]\right\}=-M_{P}^{2-D} \widetilde{T}_{\mu \nu} \delta(z), \\
& {\left[\partial^{\mu} H_{\mu \nu}-\partial_{\nu} H\right]^{\prime}-\partial^{\mu} F_{\mu \nu}+(D-2) A^{\prime} \partial_{\nu} \rho=M_{P}^{2-D} \widetilde{T}_{\mu D} \delta(z),} \\
& -\left[\partial^{\mu} \partial^{\nu} H_{\mu \nu}-\partial^{\mu} \partial_{\mu} H\right]+(D-2) A^{\prime}\left[H^{\prime}-2 \partial^{\sigma} A_{\sigma}\right]-(D-1)(D-2)\left(A^{\prime}\right)^{2} \rho=0,
\end{aligned}
$$

where $F_{\mu \nu} \equiv \partial_{\mu} A_{\nu}-\partial_{\nu} A_{\mu}$ is the $U(1)$ field strength for the graviphoton.

In terms of the component fields $H_{\mu \nu}, A_{\mu}$ and $\rho$, the full $D$-dimensional diffeomorphisms read:

$$
\begin{aligned}
& \delta H_{\mu \nu}=\partial_{\mu} \widetilde{\xi}_{\nu}+\partial_{\nu} \widetilde{\xi}_{\mu}+2 \eta_{\mu \nu} A^{\prime} \omega \\
& \delta A_{\mu}=\widetilde{\xi}_{\mu}^{\prime}+\partial_{\mu} \omega \\
& \delta \rho=2 \omega^{\prime}+2 A^{\prime} \omega
\end{aligned}
$$

where $\omega \equiv \widetilde{\xi}_{D}$. It is not difficult to check that (21) is invariant under these gauge transformations. On the other hand, the invariance of $(19)$ requires $[\omega \delta(z)]^{\prime}=0$. This then implies that $\omega(z=0)=0$. Finally, the invariance of $(20)$ can be seen to imply $\widetilde{\xi}_{\mu}^{\prime} \delta(z)=0$, which

implies $\widetilde{\xi}_{\mu}^{\prime}(z=0)=0$. Thus, the unbroken diffeomorphisms are given by (22), (23) and (24) subject to the following conditions:

$$
\begin{aligned}
& \omega(z=0)=0, \\
& \widetilde{\xi}_{\mu}^{\prime}(z=0)=0 .
\end{aligned}
$$

Note that it is the presence of a non-zero tension brane that is responsible for this reduction of gauge symmetry in the system. The condition (25) can be intuitively understood by noting that $A^{\prime}$ is discontinuous at $z=0$, so that $\omega$ must vanish at $z=0$ or else the gauge transformation (22) for $H_{\mu \nu}$ would be discontinuous. On the other hand, (26) becomes evident upon examination of $(20)$. The r.h.s. of this equation, which is given by $M_{P}^{2-D} \widetilde{T}_{\mu D} \delta(z)=A_{\mu} \tilde{f} \delta(z)$, is nothing but the mass term for the graviphoton on the brane, so that the corresponding gauge transformation on the brane should be vanishing.

Before we turn to solving the above system of equations, the following observation is in order. Note that the 1.h.s. of (20) does not contain terms with second derivatives w.r.t. $z$. This then implies that, to have a consistent solution, we must have

$$
A_{\mu}(z=0)=0
$$

In fact, this is consistent with the fact that the graviphoton does not couple to the conserved energy-momentum tensor on the brane.

\section{A. Partial Gauge Fixing}

Note that a physical solution to the above system of equations must satisfy the physical boundary conditions, namely, that all perturbations should decay to zero away from the 
brane. In particular, the graviphoton field $A_{\mu}$ must satisfy this requirement as well. It is not difficult to see that, since it vanishes on the brane, to have $A_{\mu} \rightarrow 0$ at $z \rightarrow \pm \infty$, we must assume that $A_{\mu}$ vanishes everywhere. Note however, that this actually is not an additional requirement. Indeed, we can always gauge $A_{\mu}$ away. Thus, it vanishes on the brane. On the other hand, away from the brane (that is, at $z \neq 0$ ) we can always use the unbroken diffeomorphisms (23) to set $A_{\mu}$ to zero. Thus, consider diffeomorphisms with $\omega \equiv 0$. We can then always choose $\widetilde{\xi}_{\mu}$ such that the gauge transformed $A_{\mu}$ identically vanishes. In the following we will refer to this gauge fixing as partial gauge fixing (as opposed to the complete gauge fixing which we will discuss in the next subsection).

After the aforementioned partial gauge fixing, we have the following equations of motion:

$$
\begin{aligned}
& \left\{\partial_{\sigma} \partial^{\sigma} H_{\mu \nu}+\partial_{\mu} \partial_{\nu} H-\partial_{\mu} \partial^{\sigma} H_{\sigma \nu}-\partial_{\nu} \partial^{\sigma} H_{\sigma \mu}-\eta_{\mu \nu}\left[\partial_{\sigma} \partial^{\sigma} H-\partial^{\sigma} \partial^{\rho} H_{\sigma \rho}\right]\right\}+ \\
& \left\{H_{\mu \nu}^{\prime \prime}-\eta_{\mu \nu} H^{\prime \prime}+(D-2) A^{\prime}\left[H_{\mu \nu}^{\prime}-\eta_{\mu \nu} H^{\prime}\right]\right\}+ \\
& \left\{\partial_{\mu} \partial_{\nu} \rho-\eta_{\mu \nu} \partial_{\sigma} \partial^{\sigma} \rho+\eta_{\mu \nu}\left[(D-2) A^{\prime} \rho^{\prime}+(D-1)(D-2)\left(A^{\prime}\right)^{2} \rho\right]\right\}=-M_{P}^{2-D} \widetilde{T}_{\mu \nu} \delta(z), \\
& {\left[\partial^{\mu} H_{\mu \nu}-\partial_{\nu} H\right]^{\prime}+(D-2) A^{\prime} \partial_{\nu} \rho=0} \\
& -\left[\partial^{\mu} \partial^{\nu} H_{\mu \nu}-\partial^{\mu} \partial_{\mu} H\right]+(D-2) A^{\prime} H^{\prime}-(D-1)(D-2)\left(A^{\prime}\right)^{2} \rho=0 .
\end{aligned}
$$

To solve this system of equations, it is convenient to perform the Fourier transform for the coordinates $x^{\mu}$ (we will denote the corresponding momenta via $p^{\mu}$ ), and Wick rotate to the Euclidean space (where the propagator is unique). The equations of motion for the Fourier transformed quantities read:

$$
\begin{aligned}
& -\left\{p^{2} H_{\mu \nu}+p_{\mu} p_{\nu} H-p_{\mu} p^{\sigma} H_{\sigma \nu}-p_{\nu} p^{\sigma} H_{\sigma \mu}-\eta_{\mu \nu}\left[p^{2} H-p^{\sigma} p^{\rho} H_{\sigma \rho}\right]\right\}+ \\
& \left\{H_{\mu \nu}^{\prime \prime}-\eta_{\mu \nu} H^{\prime \prime}+(D-2) A^{\prime}\left[H_{\mu \nu}^{\prime}-\eta_{\mu \nu} H^{\prime}\right]\right\}+ \\
& \left\{-p_{\mu} p_{\nu} \rho+\eta_{\mu \nu}\left[(D-2) A^{\prime} \rho^{\prime}+\rho\left(p^{2}+(D-1)(D-2)\left(A^{\prime}\right)^{2}\right)\right]\right\}=-M_{P}^{2-D} \widetilde{T}_{\mu \nu}(p) \delta(z), \\
& {\left[p^{\mu} H_{\mu \nu}-p_{\nu} H\right]^{\prime}+(D-2) A^{\prime} p_{\nu} \rho=0,} \\
& {\left[p^{\mu} p^{\nu} H_{\mu \nu}-p^{2} H\right]+(D-2) A^{\prime} H^{\prime}-(D-1)(D-2)\left(A^{\prime}\right)^{2} \rho=0 .}
\end{aligned}
$$

Let us assume that $T(p) \equiv T_{\mu}^{\mu}(p) \neq 0$. Then the most general tensor structure for the fields $H_{\mu \nu}$ and $\rho$ can be parametrized in terms of four functions $a, b, c, d$ as follows:

$$
\begin{aligned}
& \rho=M_{P}^{2-D} d T(p), \\
& H_{\mu \nu}=M_{P}^{2-D}\left\{a T_{\mu \nu}(p)+\left[b \eta_{\mu \nu}+c p_{\mu} p_{\nu}\right] T(p)\right\} .
\end{aligned}
$$

Plugging this back into the equations of motion, we obtain five equations for four unknowns $a, b, c, d$. However, one of these equations is identically satisfied once we take into account the other four (as well as the on-shell expression for $A$ ). After some straightforward computations we obtain the following system of four independent equations:

$$
\begin{aligned}
& a^{\prime \prime}+(D-2) A^{\prime} a^{\prime}-p^{2} a=-\delta(z), \\
& (D-2) A^{\prime} d=a^{\prime}+(D-2) b^{\prime}, \\
& A^{\prime}\left[(D-2) p^{2} c^{\prime}-a^{\prime}\right]=p^{2}[a+(D-2) b], \\
& a+(D-3) b-c^{\prime \prime}-(D-2) A^{\prime} c^{\prime}+d=0 .
\end{aligned}
$$


Here we note that (36) must be solved subject to the boundary conditions $a(z \rightarrow \pm \infty)=0$ (for $p^{2}>0$ ).

Note that (39) contains the second derivative of $c$ but no $\delta$-function term. This then implies that $c^{\prime}$ must be continuous at $z=0$. However, since $A^{\prime}$ is discontinuous at $z=0$, the only way that we can satisfy $(\sqrt[38]{ })$ is then to assume that $c^{\prime}$ vanishes at $z=0$. Taking into account that at $z \rightarrow \pm \infty c$ must go to zero, we can assume that $c$ identically vanishes. Then we have only three unknowns left, namely, $a, b, d$, but we still have four equations. However, one of these equations, namely, (37), is automatically satisfied once we take into account the other three equations (as well as the on-shell expression for $A$ ). In terms of the solution to (36) (which can be expressed via Bessel functions [15]), we thus have the following expression for $b$

$$
b=-\frac{1}{D-2}\left[a+\frac{1}{p^{2}} A^{\prime} a^{\prime}\right] .
$$

Note that $d=-a-(D-3) b$.

Using the above results we obtain:

$$
\begin{aligned}
& H_{\mu \nu}(p, z)=M_{P}^{2-D} a(p, z)\left\{T_{\mu \nu}(p)-\frac{1}{D-2}\left[1+\frac{1}{p^{2}} A^{\prime}(z)(\ln [a(p, z)])^{\prime}\right] \eta_{\mu \nu} T(p)\right\} \\
& \rho(p, z)=-M_{P}^{2-D} \frac{a(p, z)}{D-2}\left\{1-\frac{D-3}{p^{2}} A^{\prime}(z)(\ln [a(p, z)])^{\prime}\right\} T(p) .
\end{aligned}
$$

This solution gives the form of (linearized) gravitational interactions between matter sources localized on the brane including the tensor structure of the corresponding graviton propagator. In fact, this is a physical solution - it satisfies the physical boundary conditions at $z \rightarrow \pm \infty$, where both $H_{\mu \nu}(p, z)$ and $\rho(p, z)$ decay to zero as they should. In fact, in the following we will argue that the leading ultra-violet behavior of $H_{\mu \nu}$ and $\rho$ on the brane following from the above solution is independent of the gauge choice.

\section{B. Complete Gauge Fixing}

Recall that above we set $c$ to zero, which left us with three unknowns $a, b, d$ yet four equations. However, as we mentioned above, only three out of these four equations are independent. This gives us a hint that, before we set $c$ to zero, there was still some residual gauge symmetry left in the system. This, in fact, is indeed the case. The simplest way to see this is to note that we can actually gauge both $A_{\mu}$ and $\rho$ away. Indeed, even though $\omega$ must vanish at $z=0, \omega^{\prime}$ need not. It is then not difficult to see that we can simultaneously gauge $A_{\mu}$ and $\rho$ away.

One way to do this is to actually start from the solution (41) and (42), and find a gauge transformation that gauges $\rho$ away but does not affect $A_{\mu}$, which is vanishing. The corresponding gauge parameters are given by $(\Delta$ was defined after (8)):

$$
\begin{aligned}
\omega & =\frac{M_{P}^{2-D}}{4(D-2) \Delta} \frac{T(p)}{p^{2}}\left[2 a^{\prime} \Delta+\operatorname{sign}(z)(|z|+\Delta)\right], \\
\widetilde{\xi}_{\mu} & =\frac{M_{P}^{2-D}}{8(D-2) \Delta} \frac{i p_{\mu} T(p)}{p^{2}}\left[4 a \Delta+z^{2}+2 \Delta|z|\right],
\end{aligned}
$$


where we are working with the Fourier transformed quantities. Note that these gauge parameters formally satisfy the conditions (25) and (26). Here we note that $\widetilde{\xi}_{\mu}$ is given by the above expression up to an arbitrary additive contribution independent of $z$, which can be absorbed into $(D-1)$-dimensional diffeomorphisms (for which $\omega \equiv 0$, and $\widetilde{\xi}_{\mu}$ are independent of $z$ ).

Next, the gauge transformed field $H_{\mu \nu}$ is given by:

$$
\begin{aligned}
& H_{\mu \nu} M_{P}^{D-2}=a\left[T_{\mu \nu}(p)-\frac{1}{D-2} \eta_{\mu \nu} T(p)\right]-\frac{1}{2(D-2) \Delta} \eta_{\mu \nu} \frac{T(p)}{p^{2}}+ \\
& \frac{1}{4(D-2) \Delta} \frac{p_{\mu} p_{\nu} T(p)}{p^{2}}\left[4 a \Delta+z^{2}+2 \Delta|z|\right] .
\end{aligned}
$$

This expression has some peculiar properties. First, the last term diverges as $z \rightarrow \pm \infty$, so that $H_{\mu \nu}$ given by this expression is no longer a small perturbation around the background. Moreover, even though this term is proportional to $p_{\mu} p_{\nu}$, a probe bulk matter source, for which $p^{\mu} T_{\mu \nu}^{\text {bulk }}(p)$ need not vanish, will feel this field away from the brane. Second, even if we consider only bulk matter sources with $p^{\mu} T_{\mu \nu}^{\text {bulk }}(p)=0$, so that the last term in (45) cannot be measured, the second term in (45) is still felt by such a probe bulk matter source (unless its energy-momentum tensor is traceless). This term, however, is independent of $z$ (while the first term in (45) decays to zero at $z \rightarrow \pm \infty$ ), so there is a non-vanishing perturbation even infinitely far away form the brane. That is, the above gauge transformed solution does not satisfy the physical boundary conditions at $z \rightarrow \pm \infty$.

Another way of arriving at the above result is to use the complete gauge fixing to begin with, that is, to set $A_{\mu}$ as well as $\rho$ to zero before solving the equations of motion. This amounts to setting $d$ to zero in the system of equations (36), (37), (38) and (39). Once again, we now have three unknowns $a, b, c$ and four equations. However, as before, only three of these equations are independent. In fact, upon solving this system of equations for $a, b, c$, we obtain precisely (45) (up to terms which can be gauged away using the aforementioned $(D-1)$-dimensional diffeomorphisms).

Thus, as we see, the scalar degree of freedom $\rho$ cannot be gauged away in the sense that, if we do gauge it away, the corresponding gauge transformed solution is not physical. In particular, it does not satisfy the physical boundary conditions at $z \rightarrow \pm \infty$. However, above we saw that we cannot gauge $\rho$ away everywhere, but we could ask whether $\rho$ can be gauged away on the brane (while it is non-vanishing in the bulk) and still satisfy the physical boundary conditions.

The answer to this question is positive, but there is an important point arising in such gauge fixing that we would like to discuss here. Thus, consider a gauge transformation with the following properties:

$$
\widetilde{\xi}_{\mu}(z)=i p_{\mu} \int_{0}^{z} d z_{1} \omega\left(z_{1}\right)
$$

where $\omega(z)$ satisfies the following conditions:

$$
\omega(0)=0, \quad \omega^{\prime}(0)=\frac{1}{2(D-2)} \frac{T(p)}{p^{2}}\left[p^{2} a(p, 0)-\frac{D-3}{2 \Delta}\right]
$$


and $\omega(z)$ goes to zero at $z \rightarrow \pm \infty$ fast enough so that $\widetilde{\xi}_{\mu}(z)$ also goes to zero. Moreover, let us require that at any finite $z$ none of the quantities $\omega(z), \omega^{\prime}(z)$ and $\widetilde{\xi}_{\mu}(z)$ have poles for any values of $p^{2}$ even after we rotate back to the Minkowski space. In fact, it is non-trivial that this last condition can be satisfied. Thus, consider $\widetilde{\xi}_{\mu}(z)$ infinitesimally close to the brane. From (46) we have

$$
\widetilde{\xi}_{\mu}(z)=\frac{i p_{\mu}}{2} \omega^{\prime}(0) z^{2}+\mathcal{O}\left(z^{3}\right)
$$

On the other hand, for small $p^{2}$ we have $a(p, 0)=(D-3) / 2 \Delta p^{2}+\mathcal{O}(\Delta)$, so that $\omega^{\prime}(0)$ does not have a pole at $p^{2}=0$, nor does $\widetilde{\xi}_{\mu}(z)$ infinitesimally near the brane. The above gauge transformation then introduces no dangerous poles in $H_{\mu \nu}$ or $\rho$. Moreover, it is not difficult to see that the gauge transformed graviphoton still vanishes everywhere. The gauge transformed graviscalar $\rho$ now vanishes on the brane, but it is non-vanishing outside of the brane. Finally, the above gauge transformation does not affect the graviton components on the brane as $\omega(0)=0$ and $\widetilde{\xi}_{\mu}(0)=0$, and we have

$$
H_{\mu \nu}(p, 0) M_{P}^{D-2}=a(p, 0)\left[T_{\mu \nu}(p)-\frac{1}{D-2} \eta_{\mu \nu} T(p)\right]-\frac{1}{2(D-2) \Delta} \eta_{\mu \nu} \frac{T(p)}{p^{2}}
$$

Here we note that this result is the same as that obtained using the "brane bending" procedure of 18.19]3. In fact, the gauge transformation with the properties (46) and (47) precisely corresponds to the "brane bending" procedure, where the graviscalar component vanishes on the brane due to the fact that the "brane bending" brings us to the Gaussian normal coordinates w.r.t. the brane, where the $(D D)$ component of the metric perturbation vanishes [18,19].

There is, however, a subtlety arising in using the above gauge fixing which sets $\rho$ to zero. From the above discussion it is clear that there is no subtlety as far as the infra-red behavior of the graviton propagator is concerned. The subtlety, however, does arise in the ultra-violet. Thus, consider the variations due to the above gauge transformation in $H_{\mu \nu}$ infinitesimally near the brane:

$$
\begin{aligned}
\delta H_{\mu \nu}(z)= & -i p_{\mu} \widetilde{\xi}_{\nu}-i p_{\nu} \widetilde{\xi}_{\mu}+2 \eta_{\mu \nu} A^{\prime} \omega= \\
& p_{\mu} p_{\nu} \omega^{\prime}(0) z^{2}[1+\mathcal{O}(z)]-\eta_{\mu \nu} \frac{2}{\Delta} \omega^{\prime}(0)|z|[1+\mathcal{O}(z)] .
\end{aligned}
$$

It is not difficult to see that at large $p^{2}$, that is, for $p^{2} \Delta^{2} \gg 1$, we have $a(p, 0) \approx 1 / 2 p$, where $p \equiv \sqrt{p^{2}}$. This then implies that at large $p^{2}$ we have

$$
\omega^{\prime}(0) \approx \frac{1}{2(D-2)} \frac{T(p)}{2 p} .
$$

Thus, as we see, in (50) the term proportional to $p_{\mu} p_{\nu}$ has the following momentum structure at large $p^{2}$ :

\footnotetext{
${ }^{3}$ Some issues in the "brane bending" procedure were discussed in [20].
} 


$$
\frac{p_{\mu} p_{\nu}}{p}
$$

so that the graviton propagator blows up in the ultra-violet infinitesimally near the brane. Such a behavior is not acceptable for the graviton propagator, in particular, the corresponding perturbation is no longer small for large $p^{2}$. This implies that the diffeomorphisms required for setting $\rho$ to zero on the brane actually are not allowed.

Here, however, we would like to discuss the diffeomorphisms that do not lead to ultraviolet inconsistencies of the aforementioned type. Thus, consider diffeomorphisms where we still have (46) as well as $\omega(0)=0$, but $\omega^{\prime}(0)$ goes to zero at large $p^{2}$ as

$$
\omega^{\prime}(0) \sim \frac{1}{p^{\alpha}}
$$

(We are assuming that $\omega(z)$ is such that this gauge transformation does not introduce any infra-red problems, in particular, it does not introduce any dangerous poles.) For the gauge transformed $H_{\mu \nu}$ not to blow up at large $p^{2}$, we must then assume that $\alpha \geq 2$. Note that, if this condition is satisfied, the gauge transformation in $\rho(z=0)$ only introduces terms which are subleading compared with the leading behavior of $\rho(z=0)$ given by (42) at large $p^{2}$. That is, the allowed diffeomorphisms are such that they introduce only subleading terms into $\rho(z=0)$ at large $p^{2}$. In fact, the corresponding gauge parameter $\omega^{\prime}(0)$ vanishes in the ultra-violet limit. This fact will become important when we discuss quantum corrections on the brane.

\section{Long and Short Distance Behavior}

Let us now use (41) to obtain the tensor and momentum structures of the graviton propagator at small and large $p$. At large distance scales $r \gg \Delta$, that is, at small momenta $p \ll 1 / \Delta$, we have

$$
a(p, z=0)=\frac{1}{L p^{2}}+\ldots,
$$

where the ellipses stand for subleading corrections, and

$$
L \equiv \frac{\widehat{M}_{P}^{D-3}}{M_{P}^{D-2}}=\int d z \exp [(D-2) A]=\frac{2}{D-3} \Delta .
$$

Here $\widehat{M}_{P}$ is the $(D-1)$-dimensional (reduced) Planck scale corresponding to the $(D-1)$ dimensional Newton's constant which determines the strength of gravitational interactions mediated by the localized graviton zero mode. On the other hand, $A^{\prime}(0 \pm)=\mp 1 / \Delta$, and $a^{\prime}(p, z=0 \pm)=\mp 1 / 2$ (the latter can be seen from (36)). We therefore obtain that at small momenta

$$
H_{\mu \nu}(p, z=0) \approx \widehat{M}_{P}^{3-D} \frac{1}{p^{2}}\left[T_{\mu \nu}(p)-\frac{1}{D-3} \eta_{\mu \nu} T(p)\right]
$$

Both the momentum and tensor structures in this expression for the gravitational field on the brane are $(D-1)$-dimensional. Thus, at large distances we have the $(D-1)$-dimensional 
$1 / r^{D-4}$ Newton's law, and the number of degrees of freedom which couple to $T_{\mu \nu}$ (which can be read off the coefficient $-1 /(D-3)$ in front of the $\eta_{\mu \nu} T(p)$ term) is $(D-1)(D-4) / 2$. In particular, note that the coupling of the scalar degree of freedom $\rho \equiv \widetilde{h}_{D D}$ to brane matter is suppressed by an extra factor of order $p^{2} \Delta^{2}$ compared with that of the graviton $H_{\mu \nu}$. This can be seen from the fact that at $p^{2} \rightarrow 0$ we have $d(p, 0)=-a(p, 0)-(D-3) b(p, 0) \rightarrow$ const. $\times \Delta$ (this follows from the fact that the next-to-leading correction in (54) is $\mathcal{O}(\Delta)$ ). This then implies that

$$
\rho(p, z=0) \approx \text { const. } \times \widehat{M}_{P}^{3-D} \Delta^{2} T(p) .
$$

This, in particular, implies that at long distances we indeed have $(D-1)$-dimensional gravity. In fact, let us note that the long distance behavior of $\rho(p, z=0)$ given by (57) is gauge dependent, and it is not difficult to see that we can always go to a gauge where the coupling of $\rho(p, z=0)$ to the brane matter vanishes as $p^{2} \rightarrow 0$.

Next, let us see what happens at small distance scales $r \ll \Delta$, that is, at large momenta $p \gg 1 / \Delta$. We now have

$$
a(p, z) \approx \frac{1}{2 p} \exp (-p|z|),
$$

so that we obtain

$$
H_{\mu \nu}(p, z=0) \approx M_{P}^{2-D} \frac{1}{2 p}\left[T_{\mu \nu}(p)-\frac{1}{D-2} \eta_{\mu \nu} T(p)\right] .
$$

Both the momentum and tensor structures in this expression for the gravitational field on the brane are $D$-dimensional. Thus, at small distances we have the $D$-dimensional $1 / r^{D-3}$ Newton's law, and the number of degrees of freedom which couple to $T_{\mu \nu}$ (which can be read off the coefficient $-1 /(D-2)$ in front of the $\eta_{\mu \nu} T(p)$ term $)$ is $(D-2)(D-3) / 2=$ $(D-1)(D-4) / 2+1$. The extra degree of freedom is precisely the scalar $\rho$ which no longer decouples at large momenta. This can be seen from the fact that at large $p$ we have $d(p, 0) \approx-a(p, 0) /(D-2)$, and

$$
\rho(p, z=0) \approx M_{P}^{2-D} \frac{T(p)}{2(D-2) p} .
$$

Note that that (59) and (60) are in complete agreement with the corresponding expressions in the case of a tensionless brane embedded in the Minkowski bulk [16] as they should be. Indeed, at large $p$ the leading behavior of gravity in Randall-Sundrum brane world is expected to be the same as in the limit where the bulk vacuum energy density $\Lambda$ as well as the brane tension $f$ go to zero with the fine-tuning relation (7) fixed. In this limit we precisely have a tensionless brane in the Minkowski bulk. On the other hand, as we pointed out in the previous subsection, the allowed gauge transformations do not affect the leading behavior of $\rho(p, z=0)$ at large $p$ given by (60). That is, the coupling of $\rho$ to the brane matter is non-vanishing at large $p$, and it cannot be gauged away using the allowed diffeomorphisms. 


\section{IMPLICATIONS}

The fact that $\rho$ couples to the brane matter in the ultra-violet has important implications. Thus, note that the brane world-volume theory is not conformal as long as gravity is localized. This then implies that quantum corrections will generically generate various terms in the brane world-volume for the fields that couple to the brane matter. In the Randall-Sundrum model these fields are $H_{\mu \nu}$ and $\rho$. It is clear that the corresponding terms in the brane world-volume action should respect the $(D-1)$-dimensional diffeomorphisms on the brane (for these diffeomorphisms $\omega \equiv 0$, and $\widetilde{\xi}_{\mu}$ are independent of $z$ ). Let us confine our attention to such terms that are quadratic in $H_{\mu \nu}$ and/or $\rho$, and contain at most two derivatives w.r.t. $x^{\mu}$. Then the most general corrections of this type into the brane world-volume action are given by the following terms:

$$
\begin{aligned}
& S_{H}=\widehat{M}_{P}^{D-3} C_{1} \int_{\Sigma} d^{D-1} x\left\{\frac{1}{4}\left[\partial_{\sigma} H \partial^{\sigma} H-\partial_{\sigma} H_{\mu \nu} \partial^{\sigma} H^{\mu \nu}\right]+\frac{1}{2}\left[\partial^{\mu} H_{\mu \sigma} \partial_{\nu} H^{\nu \sigma}-\partial^{\mu} H_{\mu \nu} \partial^{\nu} H\right]\right\}, \\
& S_{\rho}=\widehat{M}_{P}^{D-3} \int_{\Sigma} d^{D-1} x\left\{C_{2}\left[\partial_{\nu} H-\partial^{\mu} H_{\mu \nu}\right] \partial^{\nu} \rho-C_{3} m_{\rho}^{2} \rho^{2}-C_{4} \partial_{\mu} \rho \partial^{\mu} \rho\right\} .
\end{aligned}
$$

The terms combined into $S_{H}$ correspond to expanding the term

$$
\widehat{M}_{P}^{D-3} C_{1} \int_{\Sigma} d^{D-1} x \sqrt{-\widehat{G}} \widehat{R}
$$

around the Minkowski background: $\widehat{G}_{\mu \nu}=\eta_{\mu \nu}+H_{\mu \nu}(\widehat{R}$ denotes the $(D-1)$-dimensional Ricci scalar constructed from the metric $\left.\widehat{G}_{\mu \nu}\right)$. Note that this term renormalizes the $(D-1)$ dimensional Planck scale, and one can show that it does not introduce any inconsistency as far as the coupling between bulk gravity and brane matter is concerned.

On the other hand, as we will point out in a moment, the terms appearing in (61) are a bit more harmful. The term with the coefficient $C_{2}$ corresponds to expanding the term

$$
\widehat{M}_{P}^{D-3} C_{2} \int_{\Sigma} d^{D-1} x \rho \sqrt{-\widehat{G}} \widehat{R}
$$

around the Minkowski background. The term proportional to $C_{3}$ (that is, the mass term for $\rho$ ) corresponds to expanding the term

$$
-\widehat{M}_{P}^{D-3} C_{3} \int_{\Sigma} d^{D-1} x \sqrt{-\widehat{G}} V(\rho)
$$

around the Minkowski background. Here $V(\rho)$ is the scalar potential for $\rho$, which is also expanded w.r.t. $\rho$. The first term in this latter expansion is given by

$$
-\widehat{M}_{P}^{D-3} C_{3} V(0) \int_{\Sigma} d^{D-1} x \sqrt{-\widehat{G}}
$$

\footnotetext{
${ }^{4}$ The fact that this term is generically generated by quantum effects on a brane was pointed out in [16, 17]. The fact that it should be included in the Randall-Sundrum model was pointed out in [21].
} 
This term renormalizes the brane tension. Then to preserve $(D-1)$-dimensional Poincaré invariance on the brane we need to accordingly fine-tune the bulk vacuum energy density. This fine-tuning is the usual fine tuning of the $(D-1)$-dimensional cosmological constant, and it will not concern us here. On the other hand, the term linear in $\rho$, which is given by

$$
-\widehat{M}_{P}^{D-3} C_{3} V_{\rho}(0) \int_{\Sigma} d^{D-1} x \rho \sqrt{-\widehat{G}},
$$

deserves a more careful consideration. Indeed, if $V_{\rho}(0) \neq 0$, this term corresponds to a tadpole for $\rho$ when expanded around the classical vacuum. It is not difficult to see that such a tadpole would destabilize the background. The reason for this is that in the presence of such a tadpole the r.h.s. of the $(D D)$ component of (3), that is, the r.h.s. of (5) now contains the delta-function source term, while its l.h.s. does not contain terms with the second derivative w.r.t. $z$. This implies that $\rho=0$ does not correspond to a consistent background. On the other hand, if $V_{\rho}$ vanishes for some other $\rho=\rho_{0}$, we must then expand around this point instead of $\rho=0$. We can then always bring the corresponding solution to the form we have been using by appropriate rescalings. The important point here, however, is that a priori there is no guarantee that the scalar potential for $\rho$ has an extremum. Thus, for instance, $V(\rho)$ could have a runaway behavior. That is, in this model we a priori have to deal with a type of the moduli problem. In the following we will, however, assume that (possibly after the aforementioned rescalings) we have $V_{\rho}(0)=0$. Finally, let us mention that the term in (61) proportional to $C_{4}$ is the kinetic term for $\rho$.

In fact, there is one more term which will be relevant for the following discussion. Thus, at the quantum level there might be generated a non-vanishing coupling of $\rho$ to the brane matter analogous to that for the graviton $H_{\mu \nu}$. That is, instead of (111) we can consider a more general coupling

$$
S_{\text {int }}=\frac{1}{2} \int_{\Sigma} d^{D-1} x T_{M N} \widetilde{h}^{M N} .
$$

Here we will assume that $T_{\mu D}=0$ as $A_{\mu}$ does not couple to the conserved energy-momentum tensor $T_{\mu \nu}$ on the brane. However, the coupling $T_{D D}$ generically need not vanish at the quantum level.

Here we note that the terms in (61) as well as the $T_{D D}$ coupling in (67) are not invariant under the diffeomorphisms given by (22), (23) and (24) subject to the conditions (25) and (26) (while the correction given by $S_{H}$ is) as in these diffeomorphisms $\omega^{\prime}(0)$ need not vanish. However, as we pointed out in the previous section, not all of these diffeomorphisms are allowed - $\omega^{\prime}(0)$ must vanish fast enough with $p^{2} \rightarrow \infty$ or else the gauge transformed graviton propagator blows up near the brane in the ultra-violet. That is, generically there is nothing preventing the terms in (61) as well as the $T_{D D}$ coupling in (67) from being generated at the quantum level. In fact, the corresponding counterterms are precisely related to ultra-violet divergences in the theory.

The trouble with the terms appearing in (61) as well as the aforementioned coupling $T_{D D}$ is that they give rise to a source term on the r.h.s. of (30), while its l.h.s. does not contain terms with the second derivative w.r.t. $z$. It is then clear that, to have a consistent solution to (30), the total source term on its r.h.s. should vanish. This, however, generically implies that we need to fine-tune parameters $C_{2}, C_{3}, C_{4}$ as well as the coupling $T_{D D}$ to have 
a continuous solution for $\rho$ and $H_{\mu \nu}$. Indeed, the values of $\rho$ and $H_{\mu \nu}$ at $z=0$ are completely determined in terms of $T_{\mu \nu}$. Note that this fine-tuning is independent of the aforementioned fine-tuning required for maintaining vanishing cosmological constant on the brane.

Note that if we naively perform the "brane bending" procedure, in the corresponding Gaussian normal coordinates $\rho$ does not couple to the brane matter, so in this gauge it might seem that the aforementioned dangerous terms are not generated. However, in the presence of (non-conformal) matter on the brane, in this gauge the brane is no longer straight but is bent w.r.t. the coordinate system that is straight w.r.t. the AdS horizon [18,19]. A priori it is then unclear how to compute quantum corrections due to the brane matter loops on such a brane, so that the conclusion that the aforementioned terms are not generated in this gauge might be incorrect. In fact, had such a conclusion been correct, we would have a puzzle - physics should certainly be independent of the gauge choice, and in the gauge where $\rho$ is non-vanishing on the brane the corresponding terms are generically expected to be generated. Note, however, that the resolution of this issue appears to be quite simple if we take into account our previous discussions - the gauge corresponding to the Gaussian normal coordinates appears to suffer from ultra-violet divergences in the graviton propagator, and is not suitable for discussing the aforementioned quantum corrections on the brane.

Thus, as we see, the fact that $\rho$ cannot be gauged away necessitates fine-tuning at the quantum level to preserve consistent coupling between bulk gravity and brane matter. A similar observation was recently made in 22] in a somewhat different context, namely, within the setup of |23] where the extra dimension has infinite volume. Because of the latter fact, however, consistent coupling between bulk gravity and brane matter can be achieved in the setup of [23] if the brane world-volume theory is conformal [22].

\section{REMARKS}

Let us now comment on quantum corrections in the bulk. In particular, as was pointed out in [24], in warped backgrounds with finite-volume non-compact extra dimensions one must be cautious about higher derivative terms in the bulk action. Note that as long as $-\Lambda \ll M_{*}^{2}$, where $M_{*}$ is the cut-off scale for higher derivative terms in the bulk action, then contributions of such terms as far as the domain wall solution is concerned are under control [24]. However, as was pointed out in [24], higher curvature terms in such warped compactifications generically lead to delocalization of gravity. Thus, inclusion of higher derivative terms of, say, the form

$$
\zeta \int d^{D} x \sqrt{-G} R^{k}
$$

into the bulk action would produce terms of the form [24

$$
\zeta \int d^{D-1} x d z \exp [(D-2 k) A] \sqrt{-\widehat{G}} \widehat{R}^{k}
$$

Assuming that $A$ goes to $-\infty$ at $z \rightarrow \pm \infty$, for large enough $k$ the factor $\exp [(D-2 k) A]$ diverges, so that at the end of the day gravity is no longer localized. 
A possible way around this difficulty might be that all the higher curvature terms should come in "topological" combinations (corresponding to Euler invariants such as the GaussBonnet term [25,26]) so that their presence does not modify the $(D-1)$-dimensional propagator for the bulk graviton modes. That is, even though such terms are multiplied by diverging powers of the warp factor, they are still harmless. One could attempt to justify the fact that higher curvature bulk terms must arise only in such combinations by the fact that otherwise the bulk theory would be inconsistent to begin with due to the presence of ghosts. However, it is not completely obvious whether it is necessary to have only such combinations to preserve unitarity. Thus, in a non-local theory such as string theory unitarity might be preserved, even though at each higher derivative order there are non-unitary terms, due to a non-trivial cancellation between an infinite tower of such terms.

We would like to end our discussion by pointing out that the aforementioned difficulty with higher curvature terms does not arise in theories with infinite-volume non-compact extra dimensions [27 30,16,21,23,22, 17]. However, consistency of the coupling between bulk gravity and brane matter might give rise to additional constraints. As we have already mentioned, in some cases the brane world-volume theory must be conformal. In such cases it would be interesting to understand if there is a relation to [31].

\section{ACKNOWLEDGMENTS}

I would like to thank Gia Dvali, Gregory Gabadadze and especially Tom Taylor for valuable discussions. Parts of this work were completed while I was visiting at Harvard University, New York University and Northeastern University. This work was supported in

part by the National Science Foundation. I would also like to thank Albert and Ribena Yu for financial support. 


\section{REFERENCES}

[1] V. Rubakov and M. Shaposhnikov, Phys. Lett. B125 (1983) 136.

[2] A. Barnaveli and O. Kancheli, Sov. J. Nucl. Phys. 52 (1990) 576.

[3] J. Polchinski, Phys. Rev. Lett. 75 (1995) 4724.

[4] P. Hor̆ava and E. Witten, Nucl. Phys. B460 (1996) 506; Nucl. Phys. B475 (1996) 94;

E. Witten, Nucl. Phys. B471 (1996) 135.

[5] J. Lykken, Phys. Rev. D54 (1996) 3693.

[6] G. Dvali and M. Shifman, Nucl. Phys. B504 (1997) 127; Phys. Lett. B396 (1997) 64.

[7] N. Arkani-Hamed, S. Dimopoulos and G. Dvali, Phys. Lett. B429 (1998) 263; Phys. Rev. D59 (1999) 086004.

[8] K.R. Dienes, E. Dudas and T. Gherghetta, Phys. Lett. B436 (1998) 55; Nucl. Phys. B537 (1999) 47; hep-ph/9807522;

Z. Kakushadze, Nucl. Phys. B548 (1999) 205; Nucl. Phys. B552 (1999) 3;

Z. Kakushadze and T.R. Taylor, Nucl. Phys. B562 (1999) 78.

[9] Z. Kakushadze, Phys. Lett. B434 (1998) 269; Nucl. Phys. B535 (1998) 311; Phys. Rev. D58 (1998) 101901.

[10] I. Antoniadis, N. Arkani-Hamed, S. Dimopoulos and G. Dvali, Phys. Lett. B436 (1998) 257.

[11] G. Shiu and S.-H.H. Tye, Phys. Rev. D58 (1998) 106007.

[12] Z. Kakushadze and S.-H.H. Tye, Nucl. Phys. B548 (1999) 180.

[13] M. Gogberashvili, hep-ph/9812296; Europhys. Lett. 49 (2000) 396.

[14] M. Visser, Phys. Lett. B159 (1985) 22;

P. van Nieuwenhuizen and N.P. Warner, Commun. Math. Phys. 99 (1985) 141.

[15] L. Randall and R. Sundrum, Phys. Rev. Lett. 83 (1999) 3370; Phys. Rev. Lett. 83 (1999) 4690.

[16] G. Dvali, G. Gabadadze and M. Porrati, Phys. Lett. B485 (2000) 208.

[17] G. Dvali and G. Gabadadze, hep-th/0008054.

[18] J. Garriga and T. Tanaka, Phys. Rev. Lett. 84 (2000) 2778.

[19] S.B. Giddings, E. Katz and L. Randall, JHEP 0003 (2000) 023.

[20] I.Ya. Aref'eva, M.G. Ivanov, W. Mueck, K.S. Viswanathan and I.V. Volovich, hepth/0004114.

[21] Z. Kakushadze, Phys. Lett. B488 (2000) 402.

[22] Z. Kakushadze, Phys. Lett. B491 (2000) 317.

[23] Z. Kakushadze, Phys. Lett. B489 (2000) 207.

[24] Z. Kakushadze, Nucl. Phys. B589 (2000) 75.

[25] B. Zwiebach, Phys. Lett. B156 (1985) 315.

[26] B. Zumino, Phys. Rept. 137 (1986) 109.

[27] R. Gregory, V.A. Rubakov and S.M. Sibiryakov, Phys. Rev. Lett. 84 (2000) 5928.

[28] C. Csaki, J. Erlich and T.J. Hollowood, Phys. Rev. Lett. 84 (2000) 5932.

[29] G. Dvali, G. Gabadadze and M. Porrati, Phys. Lett. B484 (2000) 112; Phys. Lett. B484 (2000) 129.

[30] E. Witten, hep-ph/0002297.

[31] M. Bershadsky, Z. Kakushadze and C. Vafa, Nucl. Phys. B523 (1998) 59;

Z. Kakushadze, Nucl. Phys. B529 (1998) 157; Phys. Rev. D58 (1998) 106003; Phys. Rev. D59 (1999) 045007; Nucl. Phys. B544 (1999) 265. 